\title{
Leaf-cutting ants' critical and voluntary thermal limits show complex responses to size, heating rates, hydration level, and humidity
}

\author{
Cleverson Lima ${ }^{1,3} \cdot$ André Frazão Helene $^{1} \cdot$ Agustín Camacho $^{1,2} \mathbb{D}$
}

Received: 10 September 2020 / Revised: 30 August 2021 / Accepted: 3 October 2021 / Published online: 27 November 2021

(c) The Author(s) 2021

\begin{abstract}
Thermal variation has complex effects on organisms and they respond to these effects through combined behavioral and physiological mechanisms. However, it is less clear how these traits combine in response to changes in body condition (e.g., size, hydration) and environmental factors that surround the heating process (e.g., relative humidity, start temperatures, heating rates). We tested whether these body conditions and environmental factors influence sequentially measured Voluntary Thermal Maxima ( $\mathrm{VT}_{\max }$ ) and Critical Thermal Maxima, $\left(\mathrm{CT}_{\max }\right)$ in leaf-cutting ants (Atta sexdens rubropilosa, Forel, 1908). $\mathrm{VT}_{\max }$ and $\mathrm{CT}_{\max }$ reacted differently to changes in body size and relative humidity, but exhibited similar responses to hydration level, start temperature, and heating rate. Strikingly, the $\mathrm{VT}_{\max }$ of average-sized workers was closer to their $\mathrm{CT}_{\max }$ than the $\mathrm{VT}_{\max }$ of their smaller and bigger sisters, suggesting foragers maintain normal behavior at higher temperatures than sister ants that usually perform tasks within the colony. Previous experiments based on hot plate designs might overestimate ants' $\mathrm{CT}_{\max } . \mathrm{VT}_{\max }$ and $\mathrm{CT}_{\max }$ may respond concomitantly or not to temperature rises, depending on body condition and environmental factors.
\end{abstract}

Keywords Voluntary thermal maximum $\cdot$ Body size $\cdot$ Dehydration $\cdot$ Heating rate $\cdot$ Relative humidity $\cdot$ Critical thermal maximum

\section{Introduction}

Accurately predicting ecological responses to climate change requires a thorough understanding of how organisms perform under thermal stress in different contexts. Traditionally, this problem has been approached by comparing thermal limits under different conditions that may alter tolerance levels (e.g., Angilletta et al. 2007; Christian and Morton 1992). However, since organisms integrate behavioral and

Communicated by P. Withers.

Agustín Camacho

agustin.camacho@ebd.csic.es

1 Department of Physiology, Instituto de Biociências, USP, São Paulo, SP 05508-090, Brazil

2 Department of Evolutionary Ecology, Estación Biológica de Doñana, CSIC, 26 Américo Vespucio Av., 41029 Isla de la Cartuja, Spain

3 Department of Entomology, College of Agriculture, Food, and Environment, University of Kentucky, KY 40546 Lexington, USA physiological thermal tolerance to deal with temperature rises (Williams et al. 2008), detailed information on behavioral responses to temperature is needed to accurately predict responses to climate warming.

The physiological performance of ectotherms in response to temperature change is described with a Gaussian thermal performance curve (Angilletta 2014; Camacho et al. 2018; Huey and Stevenson 1979-although often skewed from the standard original model, see Sinclair et al. 2016). Within voluntarily tolerated thermal levels, different aspects of physiological performance are optimized at different temperatures (e.g., stamina may be more optimized at lower temperatures than sprint speed, Huey et al. 1984). Nonetheless, if body temperatures rise excessively, locomotor and neural processes eventually stop, and the animals reach their Critical Thermal Maximum $\left(\mathrm{CT}_{\max }\right.$, Cowles and Bogert 1944; Jørgensen et al. 2020), which can kill them almost immediately (Angilletta et al. 2007; Christian and Morton 1992; Ribeiro et al. 2012).

At temperatures close to $\mathrm{CT}_{\max }$, animals often move away from heat sources, exhibiting their Voluntary Thermal Maximum ( $\mathrm{VT}_{\max }$, Camacho et al. 2018; Cowles and 
Bogert 1944). This trait represents how organisms try to keep their body temperatures below too costly or dangerous levels (Martin and Huey 2008). $\mathrm{VT}_{\max }$ may remain invariable across populations separated by millions of years (Wiens et al. 2019), and, despite being a behavioral trait, $\mathrm{VT}_{\max }$ has been observed to change relatively little compared with $\mathrm{CT}_{\max }$ in lizards (e.g., Camacho and Rusch 2017). Moreover, preferred temperatures correlate with $\mathrm{CT}_{\max }$ across species (Huey and Kingsolver 1993; Sinervo et al. 2010), suggesting that thermal preference and thermal tolerance may vary being positively correlated (but not always, see Huey and Bennett 1987). In contrast, $\mathrm{VT}_{\max }$ and $\mathrm{CT}_{\max }$ have been found to vary independently within populations (e.g., lizards, Camacho et al. 2018). In this sense, behavioral and physiological traits of thermal tolerance, such as $\mathrm{VT}_{\max }$ and $\mathrm{CT}_{\max }$, might respond differently to internal and external influences, such as different body conditions and environmental factors.

Physiological thermal tolerance is influenced by both environmental conditions and inherent characteristics of the organism. Among these characteristics, body size affects many physiological (Hurlbert et al. 2008; Jensen and Nielsen 1975; Ribeiro et al. 2012) and ecological traits (Johnson 2008; Kaspari 1993). Larger body size often raises the $\mathrm{CT}_{\max }$ of ectothermic animals (Angilletta et al. 2007; Christian and Morton 1992, Ribeiro et al. 2012, but see Oyen and Dillon 2018). Also, larger bodies tend to store more water and present lower rates of water loss (Edney 1977). In small arthropods (i.e., ants), longer limbs also increase the distance of the separation of the body from heated surfaces, which greatly reduces the heat load within millimeters (Cerdá and Retana 2000). Ant castes can be differentiated by morphological aspects (size), but individuals of overlapping size often specialize in different tasks within and out of the nest (called temporal castes, Wilson 1980). Accordingly, it could be expected that ants of different sizes may show different thermal tolerance as a result of exposure to different temperatures (Baudier and O'donnel 2018). Few studies have related thermal tolerance and body size in insects (e.g., Clémencet et al. 2010; Verble-Pearson et al. 2015; Baudier et al. 2015; Johnson and Stahlschmidt 2020) and none have integrated $\mathrm{VT}_{\max }$ and $\mathrm{CT}_{\max }$ before. One potential problem to explore such integration is that traditional approaches with linear models might not accurately describe the relationship of size and thermal tolerance (see Ribeiro et al. 2012 Fig. 1C, where a non-linear response of $\mathrm{CT}_{\max }$ to size is suggested by the data). Thus, the shapes of these relationships remain unknown.

The level of body hydration (HL) can be another body condition regulating thermal tolerance among ectothermic animals. Water loss rates increase with rising body temperatures (Edney 1977; Lighton and Bartholomew 1988), and dehydration stress lowers the $\mathrm{VT}_{\max }$ and $\mathrm{CT}_{\max }$ of some ectotherms (e.g., Anurans, Anderson and Andrade 2017). Small arthropods also react to water stress. Messor pergan$d e i$ ants with lower water reserves forage closer to their nests (Lighton et al. 1994), Atta Columbica ants select resources with higher water content (Bowers and Porter 1981), and individuals with specific smaller size from Atta sexdens rubropilosa are recruited to transport water to the colony when it is in hydric stress (Ribeiro and Navas 2008). Thus, dehydrated individuals might have their $\mathrm{VT}_{\max }$ lower to protect themselves from lower $\mathrm{CT}_{\max }$, especially if they are experiencing heat stress in drier environments (i.e., environments with lower relative humidity, RH). Yet, to our knowledge, such responses have not been evaluated in arthropods.

Other environmental traits are typical elements of the experimental setup. These are the temperature at which the animal starts to be heated (the start temperature, ST), and the rate at which temperature rises (the heating rate, HR, sometimes called ramping rates, Overgaard et al. 2012). Both may alter the risks of overheating by changing the time at which the animal is exposed to stressfully hot temperatures. They also can change the animal's perception of risk of overheating. For example, faster heating rates could lead an organism to seek thermal refuge at lower temperatures (i.e., present lower $\mathrm{VT}_{\max }$ ), in anticipation of a higher risk of exceeding their $\mathrm{CT}_{\max }$. In contrast, slower heating rates involving longer exposures to sublethal temperatures might induce higher physiological stress (Rezende et al. 2020). If such conditions lower an organism's $\mathrm{CT}_{\max }$, a decrease in $\mathrm{VT}_{\max }$ might be expected, under the premise that $\mathrm{VT}_{\text {max }}$ will parallel $\mathrm{CT}_{\max }$ variation. These scenarios can be tested by manipulating heating rates (HR) and start temperatures (ST) in heat tolerance experiments (Terblanche et al. 2007; Camacho et al. 2018).

Ants play indispensable roles in mediating ecosystems' services and disservices (Del Toro et al. 2012). This makes important to understand how these animals are affected by environmental changes that may be harmful, such as climate change trends. The small size and the availability of countless individuals make ants appropriate experimental models for thermal tolerance studies. Also, the leaf-cutting ant Atta sexdens rubropilosa (Forel 1908), which is the focus of this study, varies in body size both within and across castes (Hölldobler and Wilson 1990), which allowed us to test hypotheses regarding body size and thermal tolerance.

Based on the topics presented above, we tested if leafcutting ants' $\mathrm{VT}_{\max }$ and $\mathrm{CT}_{\max }$ (1) increased in parallel with body size, (2) increased or decreased similarly with both relative humidity and hydration levels, and (3) increased or decreased similarly with variations in start temperatures and heating rates.

Quantifying the effects of body conditions and environmental factors on $\mathrm{VT}_{\max }$ and $\mathrm{CT}_{\max }$ will help us better understand the integration of behavioral and physiological 
thermal tolerance. In addition, these results will inform how experimental setups influence measures of behavioral and physiological thermal tolerance.

\section{Materials and methods}

\section{Study animals}

The ants used in the experiments came from five colonies collected at Rio Claro, SP (Brazil), near the Laboratório de Formigas Urbanas (Center of Social Insect Studies, CEISUniversidade Estadual Paulista). They were maintained in laboratory at $24{ }^{\circ} \mathrm{C} \pm 1$ and $55-65 \%$ relative humidity. These colonies were brought and maintained for 1-2 years at the Laboratório de Ciências da Cognição (Department of Physiology, Instituto de Biociências, Universidade de São Paulo), where the experiments were conducted. The animals were fed every day with leaves of Acalypha spp. These conditions have been used for over 30 years by researchers of the CEIS, a reference institution of social insect studies (more details in Bueno et al. 2002 and Ribeiro et al 2012).

\section{The thermal tolerance meter}

We developed a device capable of sequentially measuring the $\mathrm{VT}_{\max }$ and $\mathrm{CT}_{\max }$ of four ants in about $15 \mathrm{~min}$, the Thermal Tolerance Meter. In this device, ants are simultaneously heated in five chambers immersed in a thermal bath. This thermal bath consists of a transparent plastic box $(15 \times 10 \times 8 \mathrm{~cm})$ filled with water $(1200 \mathrm{ml})$ and heated by a Magnetic Hot Plate Stirrer (Quimis Q261). These identical individual chambers are 5-ml polystyrene tubes, with $3.5 \mathrm{~mm}$ diameter. The chambers are horizontally inserted in the thermal bath, with the tip left outside to provide a temporal thermal refuge. The heated part of each tube has $8 \mathrm{~cm}$ length, while the refuge is $3 \mathrm{~cm}$ in length. Both openings of each tube are closed during the experiments by glass rods whose diameter fit the opening gap. Yet, they leave a very thin space which allows the thin thermocouple to pass through and record the body temperature at the model ant. Apart from closing the entrances, these rods allowed to push ants out of the thermal refuge, or the tube if necessary.

During the tests, four ants (one in each chamber) had their $\mathrm{VT}_{\max }$ and $\mathrm{CT}_{\max }$ measured in each run. Ants' body temperatures were represented by that of an individual of similar size which had been killed immediately before. For that purpose, we inserted a thermocouple in this fifth individual's thorax and placed it in the (middle) chamber after it was dead. This way, we accounted for ants' body size and shape during the measurement of $\mathrm{VT}_{\max }$ and $\mathrm{CT}_{\max }$, and avoided changing its mass, as it could have happened during freezing euthanasia. For each trial, we used a different model ant, which always had a similar size to the ants being tested. The body temperatures were monitored by a T-type thermocouple (1 mm diameter, Omega ( ) ), connected to a computer through a datalogger (Picolog® TC H8). The temperature inside the thermal refuge was monitored to assess the differences with the heated part of the chamber. The thermal dynamics and thermal heterogeneity within the heating system were assessed, and they showed satisfactorily low thermal heterogeneity (See Fig. S2 in the supplementary file https://doi.org/10.6084/m9.figshare.14414243.v2). The RH of the room ranged from 55-65\%, except during the experiments which had been designed to alter hydration level and relative humidity.

\section{Sequential measures of ants' $\mathbf{V T}_{\max }$ and $\mathrm{CT}_{\max }$ using the thermal tolerance meter}

Before each experiment, the ants were acclimated to room temperature $\left(25^{\circ} \mathrm{C}\right)$ for $1 \mathrm{~h}$ (with water ad libitum). Each individual's $\mathrm{VT}_{\max }$ was registered as the temperature at which they entered and remained in the thermal refuge for at least $7 \mathrm{~s}$. In a pilot experiment, the ants could quickly visit the thermal refuge (always less than 3-4 s). Thus, by waiting $7 \mathrm{~s}$ before registering the $\mathrm{VT}_{\max }$, we ensured the ants were avoiding the heating chamber. After recording the ants' $\mathrm{VT}_{\text {max }}$, the refuge was occupied by the glass rod, closing it and pushing each ant back to the heating chamber, preventing it to come back into the thermal refuge. In the heating part, ants kept moving inside the chamber until their legs became paralyzed, causing disorganized locomotion (which was visible for the observer because of the fully transparent thermal bath). The $\mathrm{CT}_{\max }$ was then recorded and the ants were taken to a Petri dish for cooling down and observation. The ants that did not survive for at least two hours after the experiment had their $\mathrm{VT}_{\max }$ and $\mathrm{CT}_{\max }$ disregarded in the analyses (8\% of 186 subjects tested (15) were disregarded from the analyses). At the end of each day of experiments, all tested ants were killed by decapitation and disposed.

\section{The dehydration treatment}

First, four groups of ten ants were isolated from the colonies for a few hours in recipients with water available ad libitum. Assuming that the animals were fully hydrated, they were weighted in a semi-analytical balance (readability: $0.0001 \mathrm{~g})$, which was recorded as the Initial Weight (100\% of Hydration Level). Then, the ants were placed in perforated vials (1.5 ml Eppendorf tubes, with one ant inside each), which were placed within four $7 \times 7 \times 8 \mathrm{~cm}$ sealed recipients. The bottoms of two of these recipients were filled with silica gel to create a dehydrating atmosphere within the recipients. The other two recipients had water-soaked silica gel, creating a very humid atmosphere. The vials containing the ants 
were separated from the silica by paper towels, preventing any direct contact with ether the silica or the water. After a few hours, the final weight of each animal was recorded and its Hydration Level (HL) was obtained as the difference between the initial and final weight (in \%). This procedure provided ants with a range of differently dehydrated bodies $(\sim 75-100 \%)$.

\section{Experiments}

\section{Measuring the effects of body size on $\mathrm{VT}_{\max }$ and $\mathrm{CT}_{\text {max }}$}

We measured the $\mathrm{VT}_{\max }$ and $\mathrm{CT}_{\max }$ of 49 individuals, ranging from 1.5 to $4 \mathrm{~mm}$ in head width. Head width is widely used to represent leaf-cutting ants' size (see Wilson 1980). Although some smaller individuals $(0.1-1.4 \mathrm{~mm})$ can be found outside the nest, most of them aid the larvae inside the fungal gardens. The $1.5-4 \mathrm{~mm}$ range in head width thus represents the individuals found at the foraging trails. The sizes were measured with an analogic caliper (accuracy: $0.01 \mathrm{~mm})$. For this experiment, the ST was $25^{\circ} \mathrm{C}$, but the heating rate varied on an individual basis (i.e., heating rate was calculated based on how much each ant's body temperature increased per minute during the experiment). The initial RH inside the thermal chambers during this experiment was $55-65 \%$. Potential observer bias was evaluated in the following manner: three different observers collected the measures of the $\mathrm{VT}_{\max }$ and $\mathrm{CT}_{\max }$, independently, and then compared their measures. The observed differences between their independent measures remained below one degree across the 49 measures.

\section{Measuring the effects of hydration level and relative humidity conditions on $\mathrm{VT}_{\text {max }}$ and $\mathrm{CT}_{\max }$}

The $\mathrm{VT}_{\max }$ and $\mathrm{CT}_{\max }$ of individuals with HL ranging $75-100 \%$ were tested at ambient RH of $50 \%(N=15)$ and $85 \%(N=20)$, always using individuals of about $2.3 \mathrm{~mm}$ head width, $\mathrm{ST}=25{ }^{\circ} \mathrm{C}$, and $\mathrm{HR} \sim 1{ }^{\circ} \mathrm{C} / \mathrm{min}$. The experiments were carried out in a controlled climatized room (FITOTRON EL011-Eletrolab). The initial RH of the air and inside thermal chambers was similar to the ambient $\mathrm{RH}$, as checked using a hygrometer (HT-600 Instrutherm). Measuring humidity within the thermal chambers was not possible, but even if some unnoticed variation in $\mathrm{RH}$ might happen during heating, it can be safely assumed that tubes at $50 \%$ remained always much drier than the ones at $85 \%$.

\section{Measuring the effects of start temperature and heating rates on $\mathrm{VT}_{\max }$ and $\mathrm{CT}_{\text {max }}$}

We assessed these effects in two series of trials that we later pooled together for analysis. First, we measured the $\mathrm{VT}_{\max }$ and $\mathrm{CT}_{\max }$ of 57 individuals varying the HR between 0.5 and $3{ }^{\circ} \mathrm{C} / \mathrm{min}$ but keeping the start temperature at $25^{\circ} \mathrm{C}$. Next, we measured 45 additional individuals, varying the ST between 23 and $32.5^{\circ} \mathrm{C}$, and heating rates between (0.6-2.6 $\left.{ }^{\circ} \mathrm{C} / \mathrm{min}\right)$. In this way, we ensured that ST and HR would vary independently across the full set of 102 ants, and considered both factors as continuous variables. For these trials, we used ants with about $2.3 \mathrm{~mm}$ in head width, taken directly from the colonies and kept for $1 \mathrm{~h}$ with access to water, acclimating to the room temperature $\left(25^{\circ} \mathrm{C}\right)$. The initial RH inside the thermal chambers was always in the range 55-65\%.

\section{Data analyses}

We fitted Linear Mixed Models (Bates et al. 2014) relating the $\mathrm{VT}_{\max }$ or the $\mathrm{CT}_{\text {max }}$, separately to the described predictors (body size, heating rate, start temperature, hydration level, and relative humidity conditions). In each fitted model, either the $\mathrm{VT}_{\max }$ or $\mathrm{CT}_{\max }$ was the response variable, and the corresponding predictors entered as fixed factors. Ants' colony (categorical factor with five levels) entered as grouping variable (random effect) to control the lack of independence in traits among ants of the same colony.

\section{Model selection}

Before estimating fixed and random effects, we selected the best models describing the relationships between each response trait and its most relevant predictive factors. We used the Akaike Information Criterion (AIC, Akaike 1974), which penalizes the addition of parameters more than the Bayesian Information Criterion (Wang and Liu 2006), to choose the model that best describes such relationships. One model can be considered as having a better fit than another if its AIC value is lower by a difference of at least 2 (Wang and Liu 2006). Among models with a similar fit (difference below 2 AIC units), we chose the one with the lowest AIC, but considered the result given by the second-lowest. Given the important effect of heating rates (HR), we included this factor in all the models fitted.

To flexibly identify the relationships between body size and $\mathrm{VT}_{\max }$ and $\mathrm{CT}_{\max }$ in ants, we compared four models in total, ranging from first-order equations (linear) up to a fourth-order one (non-linear). As models become more complex, having a low number of categories in the fixed factor may lead to overfitting because parameters are calculated within each level of the random effects. In this case, 
dropping terms and changing the random effect by a fixed effect (Barr et al. 2013). Thus, we repeated this model selection procedure twice, based on simpler, generalized least squares with either colony as fixed effect, or not including colony as factor. The procedure and results can be found in the supplementary material (Table S1).

When testing for the relative importance of hydration level (HL) and relative humidity (RH) on $\mathrm{VT}_{\max }$ and $\mathrm{CT}_{\max }$, we used AIC to select between four competing mixed models in which colony was again the grouping factor. These models were: (1) a model containing only HL, (2) a model including HL and RH, (3) a model including both predictive variables and HR, without interactions, and (4) a model including the three terms and an interaction between HL and RH. To describe the fit of the selected model, we calculated a conditional pseudo-Rsquared value developed for mixed models (Nakagawa et al. 2017).

When testing the effect of ST and HR on $\mathrm{VT}_{\max }$ and $\mathrm{CT}_{\text {max }}$, we selected four competing models. These models were: (1) a null model containing only the intercept, (2) a model including HR alone, (3) including HR and ST independently, and (4), a model including their interaction. We also added the conditional pseudo-Rsquared value for the selected model to describe this model's fit.

All analyses were performed in the R environment ( $R$ Development Core Team 2018) using the package nlme (Pinheiro et al. 2017). Fixed Pseudo R squared was obtained using the MuMin package (Barton 2009).

\section{Results}

\section{Effects of body size (BS) on $\mathrm{VT}_{\max }$ and $\mathrm{CT}_{\max }$}

BS did not show linear correlations with $\mathrm{VT}_{\max }(D F 42, B$ 0.130 , SD 0.294, $t 0.451, p 0.657$ ) or $\mathrm{CT}_{\max }(D F 42, B 0.185$, SD $0.369, t 0.503, p 0.621)$, although heating rate did affect both measures ( $\mathrm{VT}_{\max } ; D F 42 B 3.466$, SD 0.546, $t 6.344, p$ $0.000 ; \mathrm{CT}_{\max } ; D F 42 B 1.014$, SD: 0.368, $t 2.755, p 0.008$ ). Instead, a third-order polynomial explained the relationship between $\mathrm{VT}_{\max }$ and size better than both simpler and more complex models (AIC difference was $>2$ with second best model). The observed non-linear trend in $\mathrm{VT}_{\max }$ was created by average-sized workers (2-2.6 $\mathrm{mm}$ head width) from four different colonies, whose $\mathrm{VT}_{\max }$ reached closer to their $\mathrm{CT}_{\max }$, compared to larger and smaller individuals of the same and other colonies (Fig. 1). This trend was also indicated by the 4 th order polynom, which had the second best fit.

In turn, the $\mathrm{CT}_{\max }$ response was not better described by more complex models compared to a straight line (Table 1). These models show that both traits respond differently to body size in leaf-cutting ants. More detailed results can be

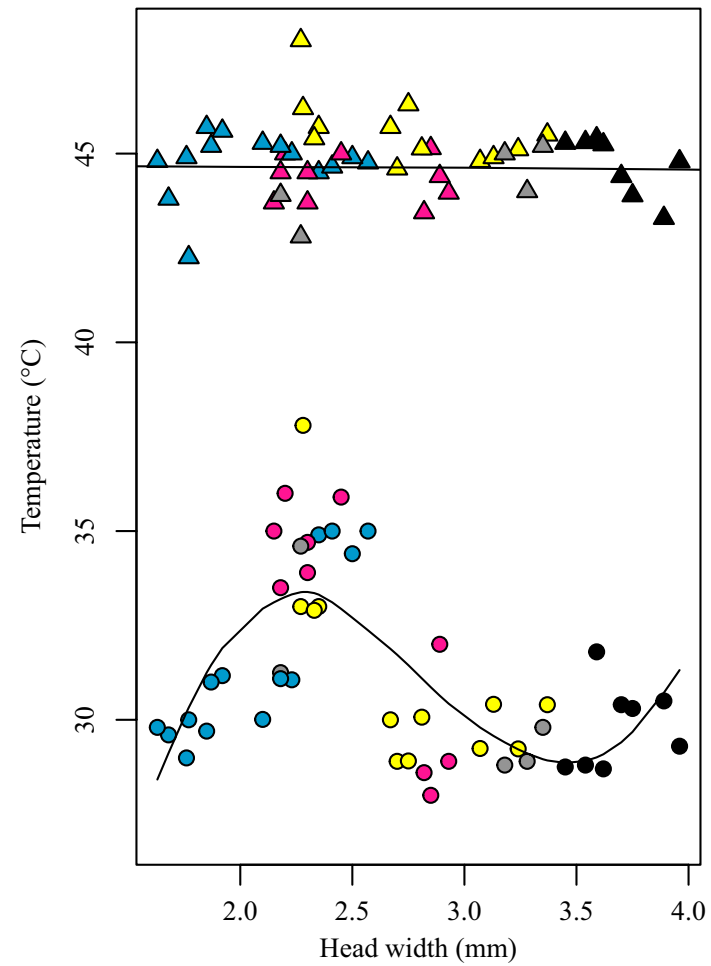

Fig. 1 Relationships between size, $\mathrm{VT}_{\max }$ and $\mathrm{CT}_{\max }$. While averagesized workers present $\mathrm{VT}_{\max }$ higher than smaller and larger workers, their $\mathrm{CT}_{\max }$ did not increase with body size. Each point in the figure represent one ant. Circles represent $\mathrm{VT}_{\max }$ and triangles represent $\mathrm{CT}_{\max }$. Color/tone indicates the colony that each ant was from. The predictive lines account for heating rates in both cases (color figure online)

Table 1 Fit of different models describing the relationship of $\mathrm{VT}_{\max }$ and $\mathrm{CT}_{\max }$ with different factors

\begin{tabular}{llll}
\hline Predictors & $D F$ & $\mathrm{VT}_{\max }$ & $\mathrm{CT}_{\max }$ \\
\hline BS linear+HR & 5 & 202.2 & 128.89 \\
BS 2nd Order + HR & 6 & 202.02 & 128.96 \\
BS 3rd Order + HR & 7 & 199.67 & 129.27 \\
BS 4th Order + HR & 8 & 201.21 & 131.19 \\
RH & 4 & 174.8 & 160.08 \\
RH+HR & 4 & 176.32 & 172.27 \\
RH+HL+HR & 6 & 159.84 & 144.46 \\
RH*HL+HR & 7 & 157.24 & 143.54 \\
Intercept & 3 & 431.64 & 336.53 \\
HR & 4 & 430.05 & 293.31 \\
HR + ST & 5 & 422.24 & 294.61 \\
HR*ST & 6 & 422.75 & 295.88 \\
\hline
\end{tabular}

$B S$ body size, $S T$ start temperature, $H R$ heating rate, $R H$ relative humidity, $H L$ hydration level, $D F$ parameters estimated

${ }^{+}$Indicates independent terms

*Indicates interaction between terms 
found in the supplementary material (Table $\mathrm{S} 1 \mathrm{~A}$ for $\mathrm{VT}_{\max }$ and $\mathrm{B}$ for $\mathrm{CT}_{\max }$ ).

\section{Combined effects of hydration level ( $\mathrm{HL})$ and relative humidity $(\mathrm{RH})$ on $\mathrm{VT}_{\max }$ and $\mathrm{CT}_{\max }$}

The competing models that we compared here were: (1) HL, (2) RH plus HL (3) the three terms, independent, (4) the three terms, with the interaction between $\mathrm{HL}$ and RH. Regarding the $\mathrm{VT}_{\max }$, model 4 was the most likely by a difference $>2$ in AIC values with the second best (Table 1). The model detects a positive but very weak interaction between $\mathrm{HL}$ and $\mathrm{RH}$, where individuals were more reactive to $\mathrm{HL}$ within the humid treatment $(N 40, B 0.106$, SD $0.050, t$ $2.110, p 0.042)$. In general, $\mathrm{VT}_{\max }$ increased with $\mathrm{HL}(N 40$, $B$ 0.079, SD: $0.035, t 2.264, p 0.031)$ and HR $(N 40, B 7.414$, SD 3.118, $t 2.377, p 0.023)$, but the effect of RH was not statistically significant $(N 40, B-8.761$, SD $3.936, t-194$, $p$ 0.061). See observations and resulting trends in Fig. 2. The full output of this model can be found in Table S2 A (Table 2).

Regarding $\mathrm{CT}_{\max }$, models 3 and 4 exhibited a similar fit (difference in AIC value $<1$ ). Both models indicated the effects of the three independent terms with no interaction (Fig. 2). Model 4 suggested a lower effect for RH, with respect to model 3 (RH: $N 40, B 1.345$, SD $0.424, t 3.1, p$ 0.003 ; HL: $N 40, B 0.081$, SD: $0.023, t 3.44, p 0.001)$, and HR ( $N 40, B$ 9.011, SD 2.069, $t 4.354, p 0.001)$. Full results can be found in the supplementary material (Table S2 B).

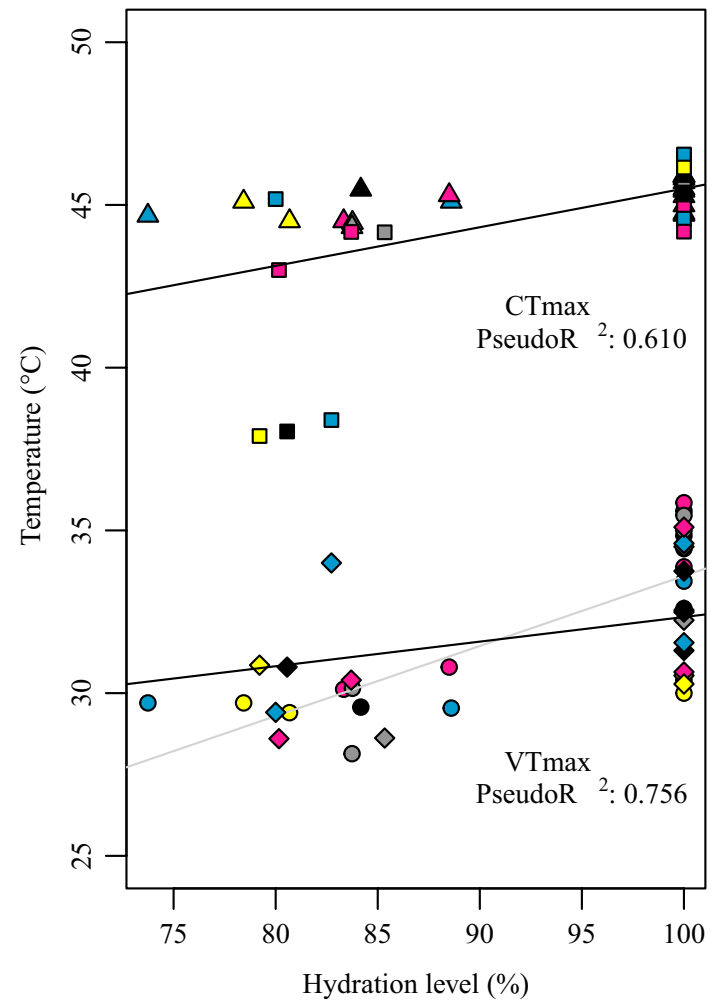

Fig. 2 Effects of hydration state and relative humidity on $\mathrm{CT}_{\max }$ (above) and $\mathrm{VT}_{\max }$ (below). Ants' $\mathrm{VT}_{\max }$ was more reactive to hydration level when heated in a humid environment ( $85 \%$ relative humidity, grey line, lozenges), compared to that of ants heated in a drier environment (50\% relative humidity, black line, circles). Meanwhile, ants' $\mathrm{CT}_{\max }$ was similarly reactive to hydration level in either a more humid ( $85 \%$ relative humidity, black line, squares) or drier environment (50\% relative humidity, triangles)
Table 2 Model coefficients table

\begin{tabular}{|c|c|c|c|c|c|c|}
\hline Trait & Effect & $D F$ & $B$ & SD & $t$ & $p$ value \\
\hline \multirow[t]{8}{*}{ VTmax } & $\mathrm{BS}$ on $\mathrm{VT}_{\max }$ & 42 & 0.13 & 0.294 & 0.451 & 0.657 \\
\hline & $\mathrm{BS}$ and $\mathrm{HR}$ on $\mathrm{VT}_{\max }$ & 42 & 3.466 & 0.546 & 6.344 & 0 \\
\hline & $\mathrm{HL}$ and $\mathrm{RH}$ on $\mathrm{VT}_{\max }$ & 40 & 0.106 & 0.05 & 2.11 & 0.042 \\
\hline & $\mathrm{HL}$ on $\mathrm{VT}_{\max }$ & 40 & 0.079 & 0.035 & 2.264 & 0.031 \\
\hline & $\mathrm{HL}$ and $\mathrm{HR}$ on $\mathrm{VT}_{\max }$ & 40 & 7.414 & 3.118 & 2.377 & 0.023 \\
\hline & $\mathrm{RH}$ on $\mathrm{VT}_{\max }$ & 40 & -8.761 & 3.936 & -194 & 0.061 \\
\hline & $\mathrm{ST}$ on $\mathrm{VT}_{\max }$ & 101 & 0.134 & 2.467 & 1.47 & 0.143 \\
\hline & $\mathrm{HR}$ on $\mathrm{VT}_{\max }$ & 101 & 1.313 & 0.373 & 3.516 & 0.001 \\
\hline \multirow[t]{7}{*}{ CTmax } & $\mathrm{BS}$ on $\mathrm{CT}_{\max }$ & 42 & 0.185 & 0.369 & 0.503 & 0.621 \\
\hline & $\mathrm{BS}$ and $\mathrm{HR}$ on $\mathrm{CT}_{\max }$ & 42 & 1.014 & 0.368 & 2.755 & 0.008 \\
\hline & $\mathrm{HL}$ on $\mathrm{CT}_{\max }$ & 40 & 0.081 & 0.023 & 3.44 & 0.001 \\
\hline & $\mathrm{HL}$ and $\mathrm{HR}$ on $\mathrm{CT}_{\max }$ & 40 & 9.011 & 2.069 & 4.354 & 0.001 \\
\hline & $\mathrm{RH}$ on $\mathrm{CT}_{\max }$ & 40 & 1.345 & 0.424 & 3.1 & 0.003 \\
\hline & $\mathrm{ST}$ on $\mathrm{CT}_{\max }$ & 101 & 0.041 & 0.048 & 0.85 & 0.396 \\
\hline & $\mathrm{HR}$ on $\mathrm{CT}_{\max }$ & 101 & 1.655 & 0.212 & 7.807 & 0 \\
\hline
\end{tabular}




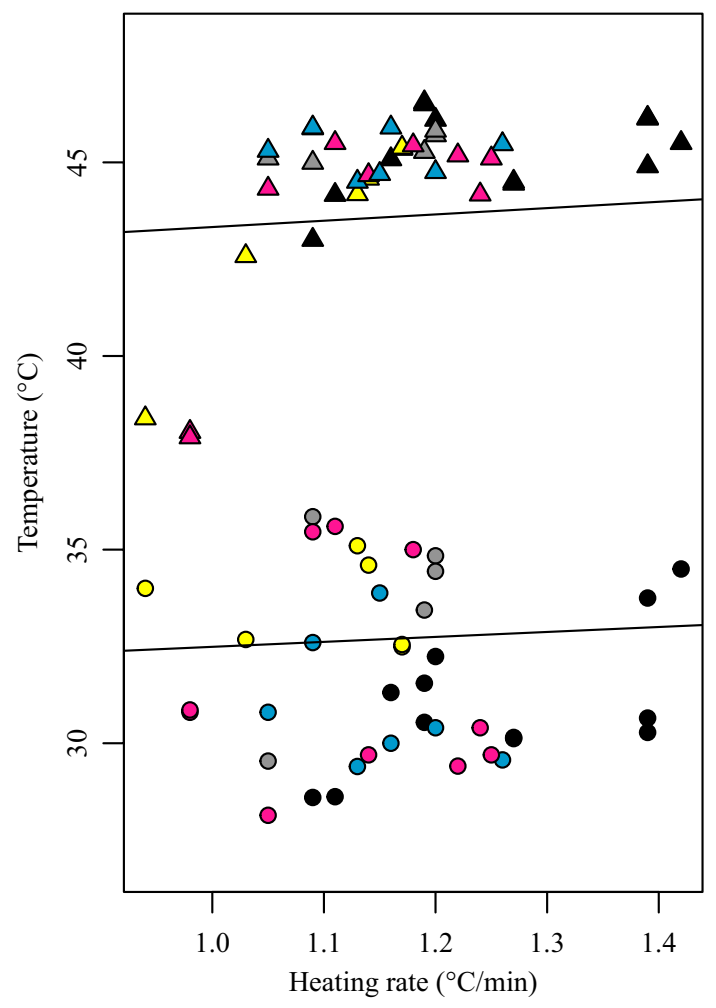

Fig. 3 Relationships between $\mathrm{VT}_{\max }, \mathrm{CT}_{\max }$ and heating rate. Heating rates increased both the $\mathrm{VT}_{\max }$ and $\mathrm{CT}_{\max }$ linearly. Each point in the figure represents one ant. Circle points represent $\mathrm{VT}_{\max }$ measures and triangle points represent $\mathrm{CT}_{\max }$ measures (color figure online)

\section{Effects of start temperature (ST) and heating rate (HR) on $V T_{\max }$ and $\mathrm{CT}_{\max }$}

The models compared were: (1), intercept, (2) HR, (3) HR plus ST and (4) HR plus ST, interacting. Among the three models with similarly low AIC (Table 1), the one with lowest AIC indicated that ST had no effects on either $\mathrm{VT}_{\max }(N$ $102, B 0.134$, SD 2.467, $t 1.47, p 0.143)$ or $\mathrm{CT}_{\max }(N 102, B$ 0.041 , SD $0.048, t 0.85, p 0.396$ ) (Fig. S1). On the contrary, raising the HR increased both ants' $\mathrm{VT}_{\text {max }}(N 102, B$ 1.313, SD 0.373, $t 3.516, p 0.001)$ and $\mathrm{CT}_{\max }(N 102, B 1.655$, SD $0.212, t 7.807, p 0.000$ ), making $\mathrm{VT}_{\max }$ range from $30{ }^{\circ} \mathrm{C}$ to $37.8{ }^{\circ} \mathrm{C}$ and $\mathrm{CT}_{\max }$ from 40.6 to $48{ }^{\circ} \mathrm{C}$ across the range of heating rates (Fig. 3, Table S3). The model including an interaction found no effects at all, but we stay with the effect of heating rate, since it was observable in all experiments.

\section{Discussion}

Our measurement system allowed us to observe how physiological and behavioral thermal tolerance may combine in response to different factors. We discovered that the $\mathrm{VT}_{\max }$ and $\mathrm{CT}_{\max }$ of ants may describe different responses (linear vs non-linear) to changes in body size. No previous data relating $\mathrm{VT}_{\max }$ and size from other ants or arthropod species are available to compare with ours. Lizards have shown a negative relationship of $\mathrm{VT}_{\max }$ with body size (Camacho et al. 2018), possibly related to age. The body size of active leafcutting ants may relate less to age and mostly to their physical caste, where ants of different sizes dedicate more time to specific tasks. Small workers (i.e., head width up to $1.4 \mathrm{~mm}$ ) often perform tasks within the nest (e.g., hyphae and larvae care, gardening, nest defense, Hölldobler and Wilson 1990; Wilson 1980) and related to water transportation (Ribeiro and Navas 2008). In turn, average-sized workers (head width about $2.2 \mathrm{~mm}$ ) are most often involved in tasks that require more time outside the nest (temporal castes, Wilson 1980). That is, they explore, forage and recruit more often (Wilson 1980). Since workers of such sizes (2-2.6 mm head width) exhibited a higher $\mathrm{VT}_{\max }$, we propose the hypothesis that within morphological castes, determined by body size, temporal castes might be formed due to their predisposition to accept higher temperatures. This seems to come at the expense of exposing themselves to higher thermal risk, since their $\mathrm{CT}_{\text {max }}$ did not increase in parallel. Future experiments, designed within a context of division of labor, may test the two hypotheses by comparing the $\mathrm{VT}_{\max }$ and thermal tolerance (e.g., $\mathrm{CT}_{\max }$ or survival time at $\mathrm{VT}_{\max }$ ) of ants specifically selected when performing different tasks. Our nests were originated from wild queens and kept at room temperature. Therefore, our studied ants were never exposed to sunrays or particularly hot ant's trails, as it would happen in the wild. In this sense, it seems unlikely that the observed increases in $\mathrm{VT}_{\max }$ arise from adaptation or acclimation due to specific exposure to heat in captivity. Therefore, it remains to be determined how some average-sized Atta ants come to be more "thermally daring".

Among hymenopterans, physiological differences are often found within morphologically defined castes (e.g., ability to follow pheromones, Robinson 2009). The separation of reproductive and working castes may be achieved by feeding the animals with different substances (Dussutour and Simpson 2009; Markin 1970; Petralia and Vinson 1978). However, no inter-castes or intra-castes differences have been reported in behavioral thermal tolerance for any hymenopteran. Yet, another example of thermally daring "special forces" might be the self-heating warrior bees of the species Apis cerana and Apis mellifera, which are able to kill Asian wasps Vespa velutina (Ken et al. 2005). Having thermally daring workers that maintain normal behavior at higher temperatures might benefit nests by extending foraging times during hotter periods or larger foraging areas, while the rest of the colony occupies cooler spaces (Cerdá and Retana 2000). More thermotolerant species have been found to be more abundant among Mediterranean species (Cerdá et al. 2002), and in at least one species, larger and 
more thermotolerant individuals forage at hotter hours of the day (Cerdá et al. 1998a, b). For leaf-cutting ants, as a consequence of spending longer times foraging at hotter temperatures, the lifespan of average-sized workers might be shortened (Mirhosseini et al. 2017; Rezende et al. 2014), compared to other workers specialized in bringing water (the smallest in the colony, Ribeiro and Navas 2008) or defending the colony (the largest ones, Powell and Clark 2004).

Our results on the $\mathrm{CT}_{\max }$ raise considerations for the design of studies of $\mathrm{CT}_{\max }$-size relationships, for leaf-cutting ants and other species. The homogeneous warming system used in this study (see Fig. S2 in the supplementary material for further details) prevented ants from creating large thermal gradients by raising over their legs to avoid the heating hotplate, where the temperature is often measured in studies on ants' thermal tolerance. The existence of these gradients in laboratory assays may explain why leaf-cutting ants' $\mathrm{CT}_{\max }$ correlates with body size in studies using a hot plate (e.g., Ribeiro et al. 2012, Whitford and Ethershank 1975), while not in ours. Longer legs might well protect larger ants by distancing them from heating surfaces (Cerdá and Retana 2000; Sommer and Wehner 2012), but our results suggest that size does not pose further protection against rapid homogenous heating, at least within leaf-cutting ants. Yet, interspecific effects of body size on ants' $\mathrm{CT}_{\max }$ have been found when using a setup more similar to ours (homogenously and slowly heated vials, Baudier et al. 2015; Baudier and O'Donnell 2020). To better understand the implications of body size on the $\mathrm{CT}_{\max }$ of ants and other arthropods, future studies might combine slow and dynamic methods for calculating the $\mathrm{CT}_{\max }$ (Lutterschmidt and Hutchison 1997). In this way, they could evaluate how behavior $\left(\mathrm{VT}_{\max }\right)$, critical limits $\left(\mathrm{CT}_{\max }\right)$ and morphology (size) interact with the time spent at stressful/sublethal temperatures (see Castañeda et al. 2015; Rezende et al. 2020).

Hot plates are widely used for estimating the $\mathrm{CT}_{\max }$ of arthropods but this technique might overestimate this trait. This methodological problem is difficult to evaluate when using large global databases of thermal tolerance data, such as the GlobTherm (Bennett et al. 2018), resulting in a potential underestimation of their vulnerability to high temperatures. For instance, Pogonomyrmex desertorum is considered one of the most thermophilic species known, with critical limits $\left(\mathrm{CT}_{\max }\right)$ up to $53{ }^{\circ} \mathrm{C}$ (Marsh 1985). In addition, other species of desert ant, heated from below (a heating mantle with a variable transformer), presented $\mathrm{CT}_{\max }>50{ }^{\circ} \mathrm{C}$ (Whitford and Ettershank 1975). Using a similar hot plate procedure, Ribeiro et al. (2012) measured $\mathrm{CT}_{\max }$ up to $53^{\circ} \mathrm{C}$ in Atta sexdens rubropilosa, which is not a thermophilic species, despite presenting $\mathrm{CT}_{\max }$ similar to P. desertorum. Meanwhile, the maximum $\mathrm{CT}_{\max }$ of $A$. sexdens rubropilosa in this study was $48{ }^{\circ} \mathrm{C}$. These cases exemplify the importance of considering the heating method during the determination of thermal tolerance in arthropods.

Hydration increased both the $\mathrm{VT}_{\max }$ and $\mathrm{CT}_{\max }$ of leafcutting ants. Both traits can also increase with HL across different ectothermic vertebrates (e.g., Anurans, Anderson and Andrade 2017; Guevara-Molina et al. 2020, and lizards, Camacho et al. 2018). Instead, the humidity had only observable effects on the $\mathrm{VT}_{\max }$ of ants. The humidity did increase the $\mathrm{CT}_{\max }$ of termites (Woon et al. 2018), suggesting that the physiological thermal tolerance of leaf-cutting ants is less susceptible to large changes in environmental humidity. We know of no previous studies comparing the strength of body condition (hydration level) and external (relative humidity) cues on voluntary maximum temperatures, but our results suggest ants may integrate both in their behavior. These responses agree with reports of water-stressed ants selecting leaves with higher water content (Bowers and Porter 1981). However, RH did not affect the $\mathrm{CT}_{\max }$ during heating, and ants' $\mathrm{VT}_{\max }$ changed most in the humid treatment. Thus, we hypothesize that this response was not anticipating higher thermal risks due to lower $\mathrm{CT}_{\text {max }}$, or risks derived from long-term exposures to high temperatures in a drier environment. Instead, they might simply be reacting to a better heat transmission within a more humid environment.

Ants' $\mathrm{VT}_{\max }$ and $\mathrm{CT}_{\max }$ were always strongly dependent on heating rate but not start temperature. Heating rates increase the $\mathrm{VT}_{\text {max }}$ of bullfrogs (Guevara-Molina et al. 2020) and some lizard species (e.g., skinks), but not of other lizards (Phrynosomatids, Camacho et al. 2018). In these cases, start temperatures only marginally affected the $\mathrm{VT}_{\max }$ of lizards, and did not affect the $\mathrm{VT}_{\max }$ of Anurans. These observations suggest that the total heating time does not affect their behavioral responses. Yet, all of them increased their $\mathrm{VT}_{\max }$ with heating rates, indicating that ants and other organisms might react to the time spent at stressful temperatures only. In the future, this could be evaluated using start temperatures closer to the $\mathrm{VT}_{\text {max }}$, and very different heating rates.

Regarding physiological thermal tolerance, heating rate may increase anurans' $\mathrm{CT}_{\max }$ while being detrimental for flies (Nyamukondiwa and Terblanche 2010). While heating rates have been often kept constant when measuring the $\mathrm{CT}_{\max }$ (Lutterschmidt and Hutchison 1997), varying them still allows detecting the effects of other variables and bringing the experiments closer to the natural conditions. In nature, heating rates should vary importantly according to where individuals are (e.g., think of a sun-hit surface compared to a nest underground), and species' ecology (e.g., terrestrial versus aquatic organisms) and physiology (e.g., more or less thermotolerant, or prone to lose water). Thus, further observations of $\mathrm{CT}_{\max }$ are needed to find general patterns.

Concluding, the $\mathrm{VT}_{\max }$ and $\mathrm{CT}_{\max }$ of leaf-cutting ants may respond differently to some factors (changes in body size, humidity) and in parallel to others (start temperatures, 
heating rates, and hydration). Our results help understanding how behavior and thermal tolerance integrate in different situations, and also show how $\mathrm{CT}_{\max }$ estimates may be affected by experimental design. We propose that leafcutting ants may have more "thermally daring" workers of average size. To continue understanding these integrative responses of organisms to temperature rises, further studies could compare the effects of these or other factors, combining dynamic and static heating systems to better understand how behavioral and physiological limits face off the temporal dimension of thermal tolerance. With few adjustments, our system could aid the observation of these limits in other small arthropods (e.g., bees, caterpillars, spiders, etc.).

Supplementary Information The online version contains supplementary material available at https://doi.org/10.1007/s00360-021-01413-6.

Acknowledgements We thank Fundação de Amparo à Pesquisa do Estado de São Paulo, Coordenação de Aperfeiçoamento de Pessoal de Nível Superior, and Marie Skłodowska-Curie Actions for funding this work and researchers. We thank Dr. Márcio Reis Custódio, Dr. Fernando Ribeiro Gomes, Maiara Moreira Macario, and Thais Carvalho Correa for their support with material and during the experiments. Also Dr. Nicholas Teets for his support with reviewing.

Author contributions CSL: Execution, methodological development, data obtention, analysis, writing. AHF: Discussion of experiments and results, infrastructural and institutional support. ACG: Conception, methodological design and development, analysis, writing.

Funding Open Access funding provided thanks to the CRUECSIC agreement with Springer Nature. This study was supported by the Fundação de Amparo à Pesquisa do Estado de São Paulo [2018/15664-5 to CSL, 12/15754-8 to AC]; Coordenação de Aperfeiçoamento de Pessoal de Nível Superior [001 to AC]; and Marie Curie Grant [897901 to AC].

Availability of data and material The raw data and supporting figures can be accessed here: https://doi.org/10.6084/m9.figshare.14414243

Code availability The raw data and supporting figures can be accessed here: https://doi.org/10.6084/m9.figshare. 14414243

\section{Declarations}

Conflict of interest The authors declare that the research was conducted in the absence of any commercial or financial relationships that could be construed as a potential competing interest.

Ethics approval According to Brazilian law, an ethics committee is not necessary for biological experiments with invertebrates.

Consent to participate and publication of data Does not apply to invertebrates as experimental subjects. All authors consent to participate in this manuscript.

Open Access This article is licensed under a Creative Commons Attribution 4.0 International License, which permits use, sharing, adaptation, distribution and reproduction in any medium or format, as long as you give appropriate credit to the original author(s) and the source, provide a link to the Creative Commons licence, and indicate if changes were made. The images or other third party material in this article are included in the article's Creative Commons licence, unless indicated otherwise in a credit line to the material. If material is not included in the article's Creative Commons licence and your intended use is not permitted by statutory regulation or exceeds the permitted use, you will need to obtain permission directly from the copyright holder. To view a copy of this licence, visit http://creativecommons.org/licenses/by/4.0/.

\section{References}

Akaike H (1974) A new look at the statistical model identification. IEEE Trans Autom Control 19:716-723

Anderson R, Andrade D (2017) Trading heat and hops for water: dehydration effects on locomotor performance, thermal limits, and thermoregulatory behavior of a terrestrial toad. Ecol Evol 7:9066-9075

Angilletta M (2014) Thermal adaptation. Oxford University Press, Oxford

Angilletta M, Wilson R, Niehaus A, Sears M, Navas C, Ribeiro P (2007) Urban physiology: city ants possess high heat tolerance. PLoS One 2:e258

Barr DJ et al (2013) Random effects structure for confirmatory hypothesis testing: keep it maximal. J Memory Lang 683:255-278

Barton K (2009) MuMIn: multi-model inference, R package version 0.12. 0. http://r-forge.r-project.org/projects/mumin/. Accessed 29 Aug 2021

Bates D, Mächler M, Bolker B, Walker S (2014) Fitting linear mixedeffects models using lme4. J Stat Softw. https://doi.org/10.18637/ jss.v067.i01

Baudier K, O'Donnell S (2018) Complex body size differences in thermal tolerance among army ant workers (Eciton burchellii parvispinum). J Therm Biol 78:277-280

Baudier KM, O'Donnell S (2020) Rain shadow effects predict population differences in thermal tolerance of leaf-cutting ant workers (Atta cephalotes). Biotropica 52(1):113-119

Baudier K, Mudd A, Erickson S, O’Donnell S (2015) Microhabitat and body size effects on heat tolerance: implications for responses to climate change (army ants: Formicidae, Ecitoninae). J Anim Ecol 84:1322-1330

Bennett J, Calosi P, Clusella-Trullas S et al (2018) GlobTherm, a global database on thermal tolerances for aquatic and terrestrial organisms. Sci Data 5:180022

Bowers M, Porter S (1981) Effect of foraging distance on water content of substrates harvested by Atta Columbica (Guerin). Ecology 62:273-275

Bueno OC, Hebling MJ, Schneider MO, Pagnocca FC (2002) Occurrence of winged forms of Atta sexdens rubropilosa Forel (Hymenoptera: Formicidae) in laboratory colonies. Neotrop Entomol 31:469-473

Camacho A, Rusch T (2017) Methods and pitfalls of measuring thermal preference and tolerance in lizards. J Therm Biol 68:63-72

Camacho A, Rusch T, Ray G, Telemeco R, Rodrigues M, Angilletta M (2018) Measuring behavioral thermal tolerance to address hot topics in ecology, evolution, and conservation. J Therm Biol 73:71-79

Castañeda LE, Rezende EL, Santos M (2015) Heat tolerance in Drosophila subobscura along a latitudinal gradient: contrasting patterns between plastic and genetic responses. Evolution 69(10):2721-2734

Cerdá X, Retana J (2000) Alternative strategies by thermophilic ants to cope with extreme heat: individual versus colony level traits. Oikos 89:155-163 
Cerdá X, Retana J, Cros S (1998a) Critical thermal limits in Mediterranean ant species: trade-off between mortality risk and foraging performance. Funct Ecol 12(1):45-55

Cerdá X, Retana J, Manzaneda A (1998b) The role of competition by dominants and temperature in the foraging of subordinate species in Mediterranean ant communities. Oecologia 117(3):404-412

Cerdá X, Dahbi A, Retana J (2002) Spatial patterns, temporal variability, and the role of multi-nest colonies in a monogynous Spanish desert ant. Ecol Entomol 27:7-15

Christian K, Morton S (1992) Extreme thermophilia in a central Australian ant, Melophorus bagoti. Physiol Zool 65:885-905

Clémencet J, Cournault L, Odent A, Doums C (2010) Worker thermal tolerance in the thermophilic ant Cataglyphis cursor (Hymenoptera, Formicidae). Insect Soc 57:11-15

Cowles R, Bogert C (1944) A Preliminary study of the thermal requirements of desert reptiles. Bull Am Mus Nat Hist 83:170-170

Del Toro I, Ribbons RR, Pelini SL (2012) The little things that run the world revisited: a review of ant-mediated ecosystem services and disservices (Hymenoptera: Formicidae). Myrmecol News 17:133-146

Dussutour A, Simpson S (2009) Communal nutrition in ants. Curr Biol 19:740-744

Edney E (1977) Water balance in land arthropods, 9th edn. Springer, Berlin

Guevara Molina EC, Gomes FR, Guerrero A (2020) Effects of dehydration on thermoregulatory behavior and thermal tolerance limits of Rana catesbeiana (Shaw, 1802). J Thermal Biol 93:102721. https://www.sciencedirect.com/science/article/pii/S030645652 0304939

Hölldobler B, Wilson EO (1990) The ants. Harvard University Press

Huey RB, Bennett AF (1987) Phylogenetic studies of coadaptation: preferred temperatures versus optimal performance temperatures of lizards. Evolution 41(5):1098-1115

Huey RB, Kingsolver JG (1993) Evolution of resistance to high temperature in ectotherms. Am Nat 142:S21-S46

Huey R, Stevenson R (1979) Integrating thermal physiology and ecology of ectotherms: a discussion of approaches. Am Zool 19:357-366

Huey RB, Bennett AF, John-Alder H, Nagy KA (1984) Locomotor capacity and foraging behaviour of Kalahari lacertid lizards. Anim Behav 32(1):41-50

Hurlbert A, Ballantyne F, Powell S (2008) Shaking a leg and hot to trot: the effects of body size and temperature on running speed in ants. Ecol Entomol 33:144-154

Jensen T, Nielsen M (1975) The influence of body size and temperature on worker ant respiration. Natura Jutlandica: 21-25. https:// www.researchgate.net/publication/280950868_The_influence_ of_body_size_and_temperature_on_worker_ant_respiration. Accessed 3 Sept 2019

Johnson R (2008) Habitat segregation based on soil texture and body size in the seed-harvester ants Pogonomyrmex rugosus and P. barbatus. Ecol Entomol 25:403-412

Johnson DJ, Stahlschmidt ZR (2020) City limits: Heat tolerance is influenced by body size and hydration state in an urban ant community. Ecol Evol 10(11):4944-4955

Jørgensen L, Robertson R, Overgaard J (2020) Neural dysfunction correlates with heat coma and $\mathrm{CT}_{\max }$ in Drosophila but does not set the boundaries for heat stress survival. J Exp Biol 223:jeb218750

Kaspari M (1993) Body size and microclimate use in Neotropical granivorous ants. Oecologia 96:500-507

Ken T, Hepburn HR, Radloff SE, Yusheng Y, Yiqiu L, Danyin Z, Neumann P (2005) Heat-balling wasps by honeybees. Naturwissenschaften 92(10):492-495

Lighton J, Bartholomew G (1988) Standard energy metabolism of a desert harvester ant, Pogonomyrmex rugosus: effects of temperature, body mass, group size, and humidity. Proc Natl Acad Sci 85:4765-4769

Lighton J, Quinlan M, Feener D (1994) Is bigger better? Water balance in the polymorphic desert harvester ant Messor pergandei. Physiol Entomol 19:325-334

Lutterschmidt WI, Hutchison VH (1997) The critical thermal maximum: history and critique. Can J Zool 75(10):1561-1574

Markin G (1970) Food distribution within laboratory colonies of the argentine ant, Tridomyrmex humilis (Mayr). Insectes Soc 17:127-157

Marsh A (1985) Aspects of the ecology of Namib Desert ants [PhD Thesis]. University of Cape Town, Faculty of Science, Department of Biological Sciences.

Martin T, Huey R (2008) Why "suboptimal" is optimal: Jensen's inequality and ectotherm thermal preferences. Am Natural 171(3):E102-E118 (pmid:18271721)

Mirhosseini MA, Fathipour Y, Reddy GV (2017) Arthropod development's response to temperature: a review and new software for modeling. Ann Entomol Soc Am 110(6):507-520

Nakagawa S, Johnson PCD, Schielzeth H (2017) The coefficient of determination $R^{2}$ and intra-class correlation coefficient from generalized linear mixed-effects models revisited and expanded. J R Soc Interface 14:20170213

Nyamukondiwa C, Terblanche JS (2010) Within-generation variation of critical thermal limits in adult Mediterranean and Natal fruit flies Ceratitis capitata and Ceratitis rosa: thermal history affects short-term responses to temperature. Physiol Entomol 35(3):255-264

Overgaard J, Kristensen TN, Sørensen JG (2012) Validity of thermal ramping assays used to assess thermal tolerance in arthropods. PLoS One 7(3):e32758

Oyen KJ, Dillon ME (2018) Critical thermal limits of bumblebees (Bombus impatiens) are marked by stereotypical behaviors and are unchanged by acclimation, age or feeding status. J Exp Biol. https://doi.org/10.1242/jeb.165589

Petralia R, Vinson S (1978) Feeding in the larvae of the imported fire ant, Solenopsis invicta: 1 behavior and morphological adaptations. Ann Entomol Soc Am 71:643-648

Pinheiro J, Bates D, DebRoy S, Sarkar D, R Core Team (2017) Linear and nonlinear mixed effects models [R package nlme version 3.1-149]. https://CRAN.R-project.org/package=nlme. Accessed 3 Feb 2018

Powell S, Clark E (2004) Combat between large derived societies: a subterranean army ant established as a predator of mature leafcutting ant colonies. Insectes Soc 51:342-351

R Core Team (2018) R: A language and environment for statistical computing. http://www.R-project.org/. Accessed 3 Feb 2018

Rezende EL, Castañeda LE, Santos M (2014) Tolerance landscapes in thermal ecology. Funct Ecol 28(4):799-809

Rezende EL, Bozinovic F, Szilágyi A, Santos M (2020) Predicting temperature mortality and selection in natural Drosophila populations. Science 369(6508):1242-1245

Ribeiro P, Navas C (2008) Colony dehydration and water collection by specialized caste in the leaf-cutting ant Atta Sexdens Rubropilosa. Journal of Insect Behavior 21:549-558

Ribeiro P, Camacho A, Navas C (2012) Considerations for assessing maximum critical temperatures in small ectothermic animals: insights from leaf-cutting ants. PLoS One 7:e32083

Robinson EJH (2009) Physiology as a caste-defining feature. Insectes Soc 56(1):1-6

Sinclair BJ, Marshall KE, Sewell MA, Levesque DL, Willett CS, Slotsbo S, Huey RB (2016) Can we predict ectotherm responses to climate change using thermal performance curves and body temperatures? Ecol Lett 19(11):1372-1385

Sinervo B, Mendez-De-La-Cruz F, Miles DB, Heulin B, Bastiaans E, Villagrán-Santa Cruz M, Sites JW (2010) Erosion of lizard 
diversity by climate change and altered thermal niches. Science 328(5980):894-899

Sommer S, Wehner R (2012) Leg allometry in ants: Extreme long-leggedness in thermophilic species. Arthropod Struct Dev 41:71-77

Terblanche J, Deere J, Clusella-Trullas S, Janion C, Chown S (2007) Critical thermal limits depend on methodological context. Proc R Soc B Biol Sci 274:2935-2943

Verble-Pearson R, Gifford M, Yanoviak S (2015) Variation in thermal tolerance of North American ants. J Therm Biol 48:65-68

Wang Y, Liu Q (2006) Comparison of Akaike information criterion (AIC) and Bayesian information criterion (BIC) in selection of stock-recruitment relationships. Fish Res 77(2):220-225

Whitford WG, Ettershank G (1975) Factors affecting foraging activity in chihuahuan desert harvester ants. Environ Entomol 4(5):689-696

Wiens J, Camacho A, Goldberg A, Jezkova T, Kaplan M, Lambert S, Miller E, Streicher J, Walls R (2019) Climate change, extinction, and Sky Island biogeography in a montane lizard. Mol Ecol 28:2610-2624

Williams S, Shoo L, Isaac J, Hoffmann A, Langham G (2008) Towards an integrated framework for assessing the vulnerability of species to climate change. PLoS Biology 6:e325

Wilson EO (1980) Caste and division of labor in leaf-cutter ants (Hymenoptera: Formicidae: Atta). Behav Ecol Sociobiol 7(2):143-156

Woon J, Boyle M, Ewers R, Chung A, Eggleton P (2018) Termite environmental tolerances are more $8 \%$ ed to desiccation than temperature in modified tropical forests. Insectes Soc 66:57-64

Publisher's Note Springer Nature remains neutral with regard to jurisdictional claims in published maps and institutional affiliations. 